\title{
Computed tomographic findings in subjects who died from respiratory disease in the National Lung Screening Trial
}

\author{
Esther Pompe ${ }^{1}$, Pim A. de Jong ${ }^{2}$, David A. Lynch ${ }^{3}$, Nikolas Lessmann ${ }^{4}$, \\ Ivana Išgum ${ }^{4}$, Bram van Ginneken ${ }^{5}$, Jan-Willem J. Lammers ${ }^{1}$ and \\ Firdaus A.A. Mohamed Hoesein ${ }^{2}$
}

Affiliations: 'Dept of Respiratory Medicine, University Medical Center Utrecht, Utrecht, The Netherlands. ${ }^{2}$ Dept of Radiology, University Medical Center Utrecht, Utrecht, The Netherlands. ${ }^{3}$ Dept of Radiology, National Jewish Health, Denver, CO, USA. ${ }^{4}$ Image Sciences Institute, University Medical Center Utrecht, Utrecht, The Netherlands. ${ }^{5}$ Dept of Radiology, Radboud University Medical Center, Nijmegen, The Netherlands.

Correspondence: Esther Pompe, University Medical Center Utrecht, Department of Respiratory Medicine, Heidelberglaan 100, P.0. Box 85500, Mailbox E.03.511, 3508GX Utrecht, The Netherlands.

E-mail: e.pompedumcutrecht.nl

@ERSpublications

Pulmonary abnormalities on CT are common in lung cancer screening and identification may add to the yield of screening http://ow.ly/qFUu308zbnC

Cite this article as: Pompe E, de Jong PA, Lynch DA, et al. Computed tomographic findings in subjects who died from respiratory disease in the National Lung Screening Trial. Eur Respir J 2017; 49: 1601814 [https://doi.org/10.1183/13993003.01814-2016]

ABSTRACT We evaluated the prevalence of significant lung abnormalities on computed tomography (CT) in patients who died from a respiratory illness other than lung cancer in the National Lung Screening Trial (NLST).

In this retrospective case-control study, NLST participants in the CT arm who died of respiratory illness other than lung cancer were matched for age, sex, pack-years and smoking status to a surviving control. A chest radiologist and a radiology resident blinded to the outcome independently scored baseline CT scans visually and qualitatively for the presence of emphysema, airway wall thickening and fibrotic lung disease. The prevalence of CT abnormalities was compared between cases and controls by using chi-squared tests.

In total, 167 participants died from a respiratory cause other than lung cancer. The prevalence of severe emphysema, airway wall thickening and fibrotic lung disease were $28.7 \%$ versus $4.8 \%, 26.9 \%$ versus $13.2 \%$ and $18.6 \%$ versus $0.5 \%$ in cases and controls, respectively. Radiological findings were significantly more prevalent in deaths compared with controls (all $\mathrm{p}<0.001$ ).

CT-diagnosed severe emphysema, airway wall thickening and fibrosis were much more common in NLST participants who died from respiratory disease, and CT may provide an additional means of identifying these diseases.

This article has supplementary material available from erj.ersjournals.com

Received: Sept 142016 | Accepted after revision: Dec 152016

Conflict of interest: Disclosures can be found alongside this article at erj.ersjournals.com

Copyright @ERS 2017 


\section{Introduction}

The National Lung Screening Trial (NLST) was the first to show that by using low-dose chest computed tomography $(\mathrm{CT})$ as a screening tool for lung cancer, a reduction in death rate from both lung cancer and death from any cause could be established [1]. Lung cancer screening has been introduced in the USA, but the debate on lung cancer screening is still continuing in Europe [2-4]. Lung cancer screening CT scans could be utilised for the detection of other diseases as well, and provide a unique opportunity to investigate the natural history and clinical relevance of certain diseases [5]; for example, calcium scores can be derived, bone density can be measured, and the radiological characteristics of chronic obstructive pulmonary disease (COPD) can be evaluated [6-8]. It is unknown whether analysing lung cancer CT scans for the presence of other (smoking-related) diseases attributes to the cost-effectiveness of lung cancer screening, but it at least provides valuable epidemiological health data [9].

Some studies have shown associations between CT diagnosis, all-cause mortality and respiratory mortality [13-16]. In most of these studies, the cause of death as reported on the death certificate was used. Almost $10 \%(175 / 1865)$ of all deaths in the CT arm of the NLST were from respiratory illnesses other than lung cancer according to the death certificates [1] and identification of these respiratory illnesses on lung cancer CT can therefore provide important information regarding the diagnosis of the disease, which might have an impact on survival. As treatment opportunities are emerging, for example, in COPD and idiopathic pulmonary fibrosis (IPF), respiratory deaths may be prevented or delayed [10-12].

We hypothesised that abnormalities in accordance with respiratory deaths should already be visible on the screening CT. NLST provides a unique opportunity to investigate the importance of these CT diagnoses for survival. The objective of this study was to evaluate the prevalence of emphysema, airway wall thickening and fibrotic lung disease in NLST participants who died of a respiratory disease in comparison with a matched control. For this purpose, we performed a retrospective case-control study in NLST participants who died from a respiratory illness other than lung cancer.

\section{Methods and materials \\ Participants}

Study design and primary results of the NLST have been reported in detail previously $[1,13]$. In brief, participants were enrolled if they were aged between 55 and 74 years and had a smoking history of more than 30 pack-years. Former smokers should not have quit more than 15 years prior to enrolment. Participants were excluded if they had a previous diagnosis of lung cancer, had undergone a chest CT within 18 months prior to enrolment, or had haemoptysis or unexplained weight loss of more than $6.5 \mathrm{~kg}$ in the previous year. From August 2002 to April 2004, 53454 participants were enrolled at 33 US medical centres, of whom 26722 were randomly assigned to screening with CT and 26732 to screening with chest radiography. Data on causes of death was retrieved on December 31, 2009. For this study, we included participants from the CT arm who died from a respiratory illness other than lung cancer, as defined on the death certificate and/or by the adjudication committee. For each case, a participant who was still alive at the most recent follow-up (6.5 years after randomisation) was matched based on (in order of importance): smoking status, sex, age and pack-years. Matching of the participants was performed automatically using software developed in-house. If there were multiple matches, one of them was chosen randomly.

At the beginning of the trial, all participants had to fill in a questionnaire on whether they had diagnoses of other illnesses prior to the study. This diagnosis was used to evaluate whether our CT findings were novel or not.

\section{Causes of death}

Cause of death was detected based on information derived from family members or friends through administration of annual (lung screening study) or semi-annual (American College of Radiology Imaging Network) health and vital status information forms or through other information, for example, obituaries [14]. The death certificate was obtained and sent to a nosologist for coding. Causes of death were coded using the ICD-10 coding system.

\section{CT acquisition}

All participants underwent low-dose chest CT scanning with a standard protocol that was developed by medical physicists associated with the trial [15]. All CT scans were acquired using multidetector scanners. Participants were scanned with $120 \mathrm{kVP}$ or $140 \mathrm{kVP}$, depending on their weight. The average effective dose was approximately $1.5 \mathrm{mSv}$. No intravenous contrast was administered and no ECG synchronisation was performed.

\section{Chest $\mathrm{CT}$ reading}

A board-certified chest radiologist and a radiology resident, blinded to the outcome, independently scored baseline CT scans for the presence of centrilobular emphysema (CLE) (subclassified as mild, moderate, or 
severe (confluent CLE or advanced destructive emphysema)) [15], airway wall thickening (absent or present), fibrotic lung disease (absent or present) or no pulmonary abnormalities, i.e. normal lungs. Normal lungs could contain nodules. Examples of CT abnormalities are shown in figures 1-3, and were defined according to the Fleischner Society glossary [15]. Airway remodelling was subjectively defined as thicker airway walls than normal, as there is no strict definition. Interstitial lung abnormalities were defined as changes affecting $>5 \%$ of the lung and could include reticular or ground glass abnormalities, centrilobular nodularity, cysts, honeycombing, or traction bronchiectasis [16]. Subsequently, fibrotic lung disease was classified as fibrotic nonspecific interstitial pneumonia (NSIP), usual interstitial pneumonia (UIP), or other/indeterminate [16]. CT scoring took approximately $1 \mathrm{~min}$, including loading of the images and reporting the findings in a digital database. Discordant cases were resolved by a consensus reading.

\section{Statistical analysis}

Normally distributed results are presented as mean \pm SD values. Non-normally distributed data are presented as median (interquartile range) values. Normally distributed data were compared between cases and controls using t-tests, whereas non-normally distributed data were compared using Mann-Whitney U-tests. The prevalence of CT abnormalities was compared using Chi-squared tests.

Because the cause of death on a death certificate can be inaccurate, the NLST used a review committee (Endpoint Verification Process; EVP) to assign the underlying cause of death. In the original New England Journal of Medicine publication, the death certificates and not the EVP diagnoses were published. We re-analysed our data after evaluation by the EVP in order to provide precise prevalence numbers and to evaluate the importance of the EVP. The process of evaluation by the EVP is described in the supplementary material.

Inter-observer agreement for the emphysema scores between the two observers was calculated using weighted kappa statistics, and presence or absence of other pulmonary abnormalities was also calculated using $\kappa$-values. Reliability was rated poor for $\kappa$-values of $<0.20$, fair for $\kappa$-values between $0.21-0.40$, moderate for $\kappa$-values of $0.41-0.60$, good for $\kappa$-values of $0.61-0.80$ and excellent for $\kappa$-values of $>0.80$. All p-values lower than 0.05 were considered statistically significant. Statistical analyses were performed with SPSS version 23.0 (SPSS, Chicago, IL, USA).

\section{Results}

Of the 172 deaths and controls, five deaths turned out be from lung cancer, as defined by the EVP. Therefore, we excluded five cases and five controls prior to analysis, resulting in 167 deaths and controls.
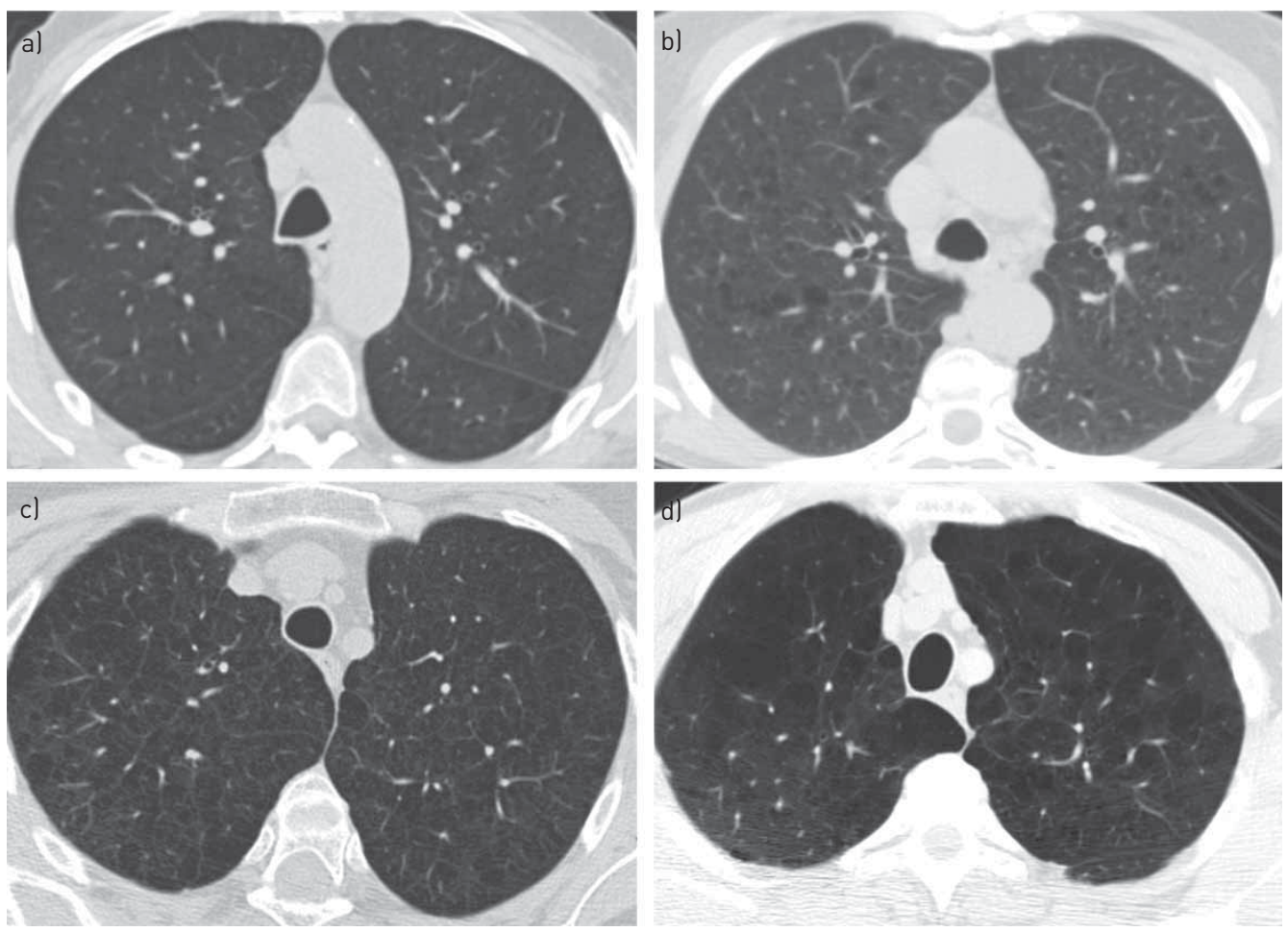

FIGURE 1 Examples of several stages of emphysema. a) Normal lungs; b) mild centrilobular emphysema; c) moderate centrilobular emphysema; and d) severe centrilobular emphysema. 


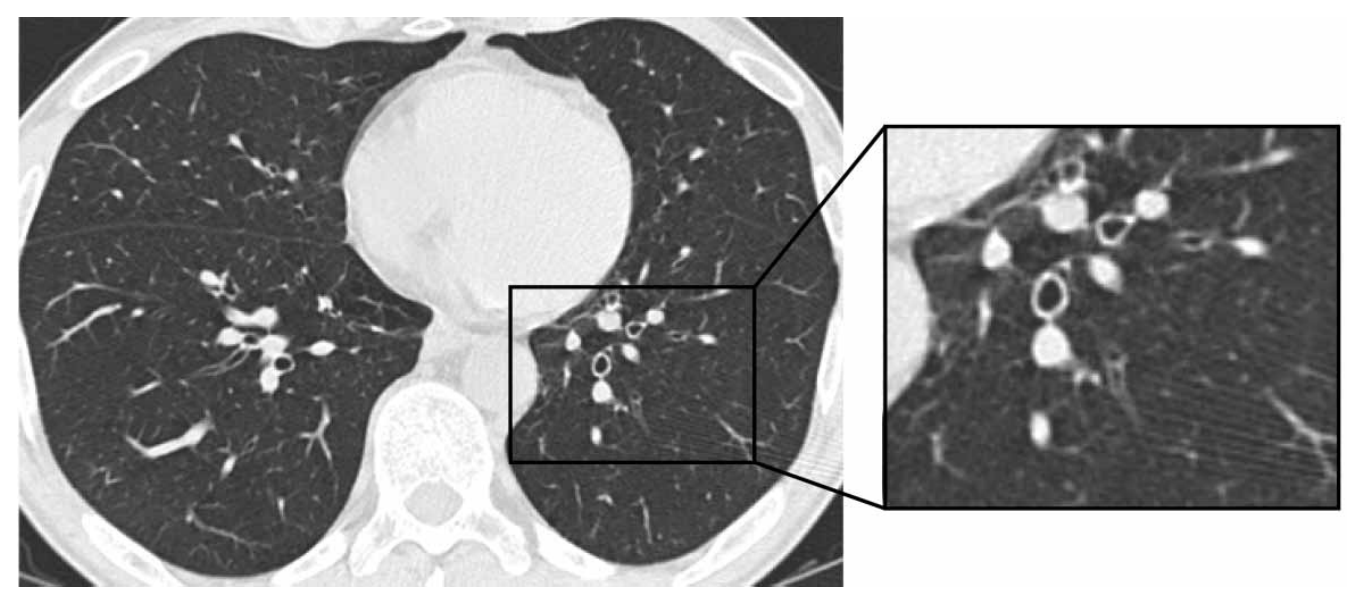

FIGURE 2 Example of airway wall thickening in axial view, with magnification of a part with thickened airways.

Causes of death according to the death certificate are shown in table 1. Seven cases and three controls had lung cancer at the time of death adjudication, but they had not died from lung cancer. Demographics and the prevalence of radiological lung disease of the 167 deaths and controls are presented in table 2. Severe emphysema was more prevalent in participants who died compared to participants still alive (28.7\% versus $4.8 \% ; \mathrm{p}<0.001$ ), whereas the prevalence of mild and moderate emphysema did not differ significantly between cases and controls. Fibrotic lung disease was present in 32 (9.6\%) participants, of whom 31 had died. One subject who was still alive had NSIP with fibrosis. Among the 31 participants who died with fibrotic lung disease the following radiological patterns were observed; 11 had UIP, 16 had NSIP with fibrosis, one had end-stage Langerhans cell histiocytosis, one had possible asbestosis, one had pleuroparenchymal fibroelastosis and one had indeterminate fibrotic lung disease. The presence of airway remodelling was more prevalent in participants who died compared with participants still alive (26.9\% versus 13.2\%). Data on overlap between visual scores are shown in figure S1. The kappa values for the CT diagnoses between the two readers were moderate to excellent (severe emphysema (weighted kappa): 0.910; airway remodelling: 0.702; fibrotic lung disease: 0.843; normal lungs: 0.794).

The CT findings of our cases were compared with the existence of a prior diagnosis of lung disease as derived from questionnaires. Of the 48 cases who had severe emphysema, 24 (50\%) were known to have COPD and/or emphysema and six (13\%) of them were known to have adult asthma. Of the 45 cases with airway remodelling, 17 (38\%) were known to have COPD and/or chronic bronchitis, and nine (20\%) were known to have adult asthma. Of the 31 cases with fibrotic lung disease, none had a prior diagnosis of fibrosis of the lungs, but two (6\%) were known to have asbestosis.

\section{EVP evaluation}

In 55 participants for whom the EVP agreed with the death certificate, the prevalence of radiological lung disease remained higher in participants who died compared with participants who were still alive (table 3 ).

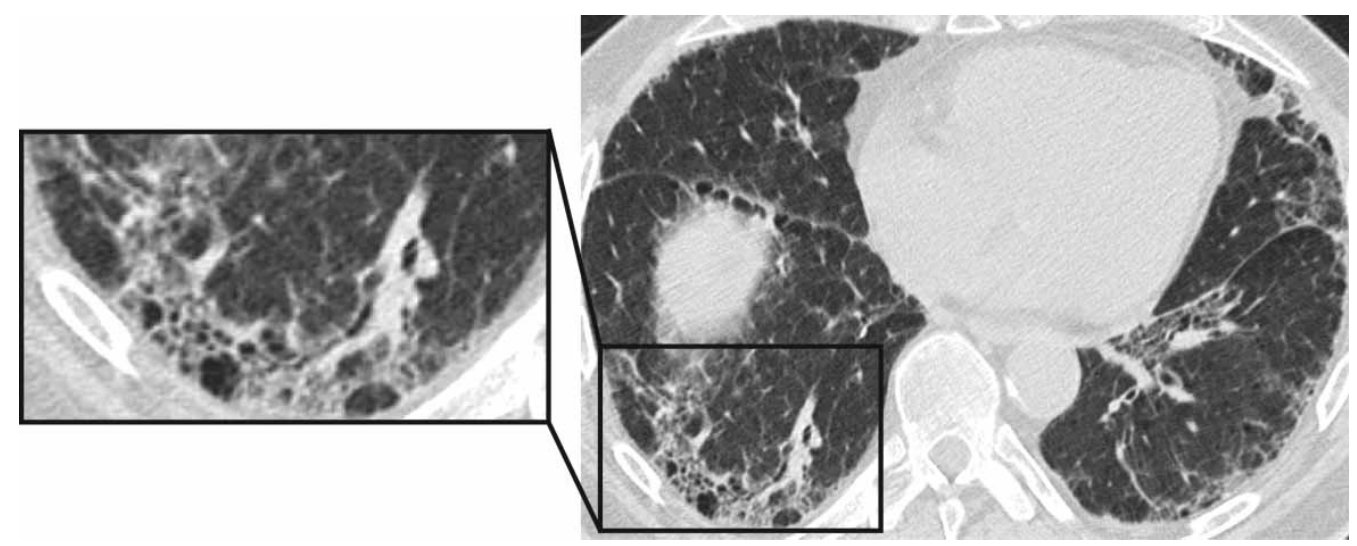

FIGURE 3 Example of fibrotic lung disease with magnification of a diseased part of the lung. 
TABLE 1 Causes of death of 167 cases, according to the death certificate

\begin{tabular}{|c|c|c|}
\hline ICD code & Cause of death & n (\%) \\
\hline J110 & Influenza due to unidentified influenza virus with pneumonia & $1(0.3)$ \\
\hline J128 & Pneumonia due to coxsackievirus & $1(0.3)$ \\
\hline J151 & Pneumonia due to Pseudomonas & $1(0.3)$ \\
\hline J180 & Bronchopneumonia, unspecified organism & $1(0.3)$ \\
\hline J189 & Pneumonia & $17(5.1)$ \\
\hline J439 & Emphysema & $10(3.0)$ \\
\hline $\mathrm{J} 440$ & COPD with acute lower respiratory infection & $5(1.5)$ \\
\hline $\mathrm{J} 441$ & Acute exacerbation & $2(0.6)$ \\
\hline J448 & Other chronic obstructive pulmonary disease & $3(0.9)$ \\
\hline J449 & COPD & $91(27.2)$ \\
\hline J459 & Unspecified asthma & $1(0.3)$ \\
\hline J690 & Aspiration pneumonia & $3(0.9)$ \\
\hline J80X & Acute respiratory distress syndrome & $1(0.3)$ \\
\hline J841 & Alveolar proteinosis & $16(4.8)$ \\
\hline$J 849$ & Interstitial pulmonary disease, unspecified & $5(1.5)$ \\
\hline J969 & Respiratory failure, unspecified & $4(1.2)$ \\
\hline J984 & Other disorders of the lung & 5 (1.5) \\
\hline
\end{tabular}

COPD: chronic obstructive pulmonary disease.

Prevalence numbers of emphysema and airway remodelling were higher in the group in which the EVP verified a respiratory cause of death compared with the group in which the cause of death was based on the death certificate only ( $43.6 \%$ versus $29.7 \%$ for emphysema and $40.0 \%$ versus $26.7 \%$ for airway remodelling). In line with the previous group, the presence of severe emphysema, but not the presence of mild and moderate emphysema, was significantly different between cases and controls $(\mathrm{p}<0.001)$. In 26 participants who died from a respiratory illness according to the death certificate, the EVP did not agree. In this group, 16 (61.5\%) participants died from COPD according to the certificate, but causes of death as reported by the EVP were mainly described as being a circulatory disease or a neoplasm. In these 26 participants, the prevalence of radiological lung disease was not significantly different from that of the matched controls (table 4).

\section{Discussion}

With the implementation of annual lung cancer screening in the USA in former and current smokers, up to 10 million individuals will be eligible for screening in the USA [17]. Our study shows that lung cancer screening scans contain important health information beyond that for lung cancer. By evaluating participants who died from respiratory causes other than lung cancer, we showed that some CT diagnoses were much more common in participants who died from a respiratory cause. Further research is needed to investigate whether evaluation of CT scans for other abnormalities is able to prevent deaths from respiratory diseases in addition to lung cancer.

TABLE 2 Demographics and prevalence of radiological disease on chest computed tomography stratified by mortality due to a respiratory illness, based on the death certificate

\begin{tabular}{lccc} 
Variable & Alive & Died of respiratory illness & p-value \\
\hline Subjects & 167 & 167 & \\
Age years & $64.9 \pm 5.7$ & $65.0 \pm 5.8$ & 0.93 \\
Male sex & $111(66.5)$ & $111(66.5)$ & 0.52 \\
Current smoker & $93(55.7)$ & $92(55.0)$ & 0.50 \\
Pack-years & $60.0(47.3-84.0)$ & $61.0(47.0-86.0)$ & 0.69 \\
Emphysema & $48(28.7)$ & & \\
$\quad$ Mild & $24(14.3)$ & $35(21.0)$ & 0.06 \\
$\quad$ Moderate & $8(4.8)$ & $35(21.0)$ & 0.08 \\
$\quad$ Severe & $1(0.5)$ & $48(28.7)$ & $<0.001$ \\
Fibrotic lung disease & $22(13.2)$ & $31(18.6)$ & $<0.001$ \\
Airway remodelling & $58(34.7)$ & $15(26.9)$ & 0.001 \\
Normal lungs & & & $<0.001$ \\
\hline
\end{tabular}

Data are presented as $\mathrm{n}, \mathrm{n}(\%)$, mean \pm SD or median (interquartile range). 
TABLE 3 Demographics and prevalence of radiological disease on chest computed tomography stratified by mortality due to a respiratory illness, based on the death certificate and confirmed by the Endpoint Verification Process

\begin{tabular}{lccc} 
& Alive & Died & p-value \\
\hline Subjects & 55 & 55 & \\
Age years & $63.0 \pm 5.5$ & $65.3 \pm 5.8$ & 0.04 \\
Male sex & $37(67.3)$ & $37(67.3)$ & 0.58 \\
Current smoker & $34(61.8)$ & $34(61.8)$ & 0.58 \\
Pack-years & $54.0(43.0-84.0)$ & $61.5(49.5-98.0)$ & 0.20 \\
Emphysema & $14(25.5)$ & $9(16.4)$ & 0.35 \\
$\quad$ Mild & $8(14.5)$ & $13(23.6)$ & 0.33 \\
$\quad$ Moderate & $2(3.6)$ & $24(43.6)$ & $<0.001$ \\
$\quad 1(1.8)$ & $7(12.7)$ & 0.03 \\
Fibrotic lung disease & $9(16.4)$ & $22(40.0)$ & 0.01 \\
Airway remodelling & $42(76.4)$ & $15(27.3)$ & $<0.001$ \\
Normal lung & & & \\
\hline
\end{tabular}

Data are presented as $\mathrm{n}, \mathrm{n}(\%)$, mean $\pm \mathrm{SD}$ or median (interquartile range).

Given the substantial costs of CT lung cancer screening [7], rapid reading $(<1 \mathrm{~min})$ of the CT scans can provide additional information by identifying diagnoses leading to respiratory mortality. In this study, we showed that the presence of fibrosis, severe emphysema and airway remodelling were strongly associated with respiratory mortality. As $10 \%$ of the deaths found in the NLST were a result of respiratory causes other than lung cancer, CT diagnoses could be helpful in the identification of the underlying entities, if not diagnosed or treated previously. Additionally, it has been shown that systematic identification of emphysema and lung fibrosis during lung cancer screening is important because both have been shown to be associated with an increased risk of lung cancer $[18,19]$.

With the identification of pulmonary abnormalities on CT, in addition to lung cancer, the yield of lung cancer screening might improve by treating subject with these "incidental" findings. Of the participants we identified with severe emphysema, 50\% did not have a prior diagnosis of emphysema or COPD. In addition, 29 out of 31 participants we found to have fibrotic changes did not have a prior history of fibrotic lung disease. Thus, identifying these abnormalities could have an impact on survival in participants who are at risk of dying from a respiratory cause.

In evaluating cause-specific mortality, the cause of death is mostly based on the information provided by the death registry. Although this information is readily available, it has been shown that the cause of death

TABLE 4 Demographics and prevalence of radiological disease on chest computed tomography (CT) stratified by mortality of participants where the Endpoint Verification Process did not confirm a respiratory cause of death other than lung cancer as reported by the death certificate

\begin{tabular}{lccc} 
& Alive & Died & p-value \\
\hline Subjects & 26 & 26 & \\
Age years & $63.8 \pm 5.4$ & $64.4 \pm 5.7$ & 0.71 \\
Male sex & $6(23.1)$ & $6(23.1)$ & 0.63 \\
Current smoker & $14(53.8)$ & $14(53.8)$ & 0.61 \\
Pack-years & $62.5(44.4-85.0)$ & $64.5(43.6-81.0)$ & 1.0 \\
Emphysema & $9(34.6)$ & $7(26.9)$ & 0.76 \\
$\quad$ Mild & $6(23.1)$ & $3(11.5)$ & 0.23 \\
$\quad$ Moderate & $1(3.8)$ & $7(26.9)$ & 0.05 \\
$\quad 1(3.8)$ & $3(11.5)$ & 0.61 \\
Severe & $7(26.9)$ & $6(23.1)$ & 1.0 \\
Airrotic lung disease & $16(61.5)$ & $12(46.2)$ & 0.40 \\
Normal lung & & & \\
\hline
\end{tabular}

Data are presented as $\mathrm{n}, \mathrm{n}(\%)$, mean \pm SD or median (interquartile range). 
as described is not always correct $[14,20]$. As a result, analyses that are based on these data might be under- or overestimating a real effect. In the data we analysed, the cause of death derived from the death certificate was modified in $53.8 \%$, which certifies the importance of the EVP.

When evaluating participants who had died from a respiratory cause other than lung cancer, as defined by the death certificate, the presence of fibrosis, severe emphysema and airway remodelling were all more prevalent in participants who had died. By presence, the presence of normal lung findings was more common in participants that were still alive. In participants for whom the EVP did not confirm a respiratory cause of death, there were no associations between CT diagnosis and mortality, although this could be due to limited power. For severe emphysema, we observed a trend and for fibrotic lung disease the same direction of effect.

Previous studies have shown that the presence of emphysema as derived from quantitative CT is associated with both all-cause and respiratory mortality [21, 22]. Although automatic emphysema quantification is feasible when evaluating a large number of scans, the challenge in quantitative emphysema measures is that absolute values differ between scanners, radiation dose, filters and other confounders. At present, there is no correction factor and nor single threshold for emphysema. The same holds for measuring airway wall thickness, which can be affected by the level of inspiration and image quality. In addition to the variability in automatic measurements, automatic calculations may take some time and may also require manual adjustments. Although segmentation of the lungs and airways can be done within a minute, calculating airway wall thickness takes somewhat longer (up to $5 \mathrm{~min}$ ). In addition, automatic identification of fibrotic changes remains a challenge. Hence, to be able to identify emphysema and airway remodelling, as well as the presence of fibrosis, visual evaluation remains a good alternative that, as we have shown, can be done fairly rapidly with good observer agreement.

Although the presence of mild and moderate emphysema has been shown to be of clinical relevance in terms of respiratory impairment [23], only the presence of severe emphysema as determined by quantitative CT has previously been shown to be associated with "short-term" mortality $[21,22,24]$. The results in this cohort, which are based on visual evaluation, confirm these results. Even in the group for which the adjudication committee did not confirm a respiratory cause of death, the prevalence of severe emphysema was higher in participants who died. Albeit not statistically significant, these results may support the findings of Haruna et al. [21] and JOHANNESSEN et al. [22], who found an association between severe emphysema and other non-respiratory causes of death (cardiovascular and cancer) as well.

A strength of this study was the ability to study a relatively large number of respiratory deaths in screened participants, with verified causes of death, given the sample size of NLST. Other studies that evaluated pulmonary CT abnormalities and mortality all used information acquired from death registries, which might lead to an underestimation of the actual effect. We were able to analyse participants in which the EVP did not confirm a respiratory cause of death, and showed that lung abnormalities on CT are specifically associated with respiratory mortality as defined by the EVP, which verifies the importance of an adjudication committee.

Some limitations need to be addressed. First, because the results were stratified by causes of death as defined by the EVP and death certificate, the number of participants in the subgroups was limited. Secondly, because the EVP systematically selected COPD deaths, but not lung fibrosis or other respiratory deaths, the subgroups analysed in this study might have been influenced by selection bias. Although there was still a clear difference in the presence of fibrosis between cases and controls, this effect might be even larger if a broader population was to be included. Thirdly, visual CT reading will inevitably involve inter-observer variation. Although we provided results on good agreement between the two observers, future studies should focus on how agreement among different readers and centres could be optimised, in order to provide the best form of this rapid reading method.

In conclusion, we showed that rapid reading of lung cancer screening CT scans for significant pulmonary abnormalities can provide diagnoses that are much more common in participants who died of a respiratory cause. Eventually it may even increase the yield of lung cancer screening by possibly reducing the number of respiratory deaths other than lung cancer.

\section{Acknowledgements}

The authors thank the National Cancer Institute for access to NCI data collected by the National Lung Screening Trial. The statements contained herein are solely those of the authors and do not represent of imply concurrence or endorsement by NCI.

\section{References}

1 National Lung Screening Trial Team,Aberle DR, Adams AM, et al. Reduced lung-cancer mortality with low-dose computed tomography screening. N Engl J Med 2011; 365: 395-409. 
2 Field JK, van Klaveren R, Pedersen JH, et al. European randomized lung cancer screening trials: Post NLST. J Surg Oncol 2013; 108: 280-286.

3 Shlomi D, Ben-Avi R, Balmor GR, et al. Screening for lung cancer: time for large-scale screening by chest computed tomography. ERJ 2014; 44: 217-238.

4 Kauczor H-U, Bonomo L, Gaga M, et al. ESR/ERS white paper on lung cancer screening. ERJ 2015; 46: 28-39.

5 Mets OM, de Jong PA, Prokop M. Computed tomographic screening for lung cancer: an opportunity to evaluate other diseases. JAMA 2012; 308: 1433-1434.

6 Jairam PM, de Jong PA, Mali WPTHM, et al. Impact of cardiovascular calcifications on the detrimental effect of continued smoking on cardiovascular risk in male lung cancer screening participants. PLoS One 2013; 8: e66484.

7 Buckens CF, van der Graaf Y, Verkooijen HM, et al. Osteoporosis markers on low-dose lung cancer screening chest computed tomography scans predict all-cause mortality. Eur Radiol 2015; 25: 132-139.

8 Mets OM, Buckens CFM, Zanen P, et al. Identification of chronic obstructive pulmonary disease in lung cancer screening computed tomographic scans. JAMA 2011; 306: 1775-1781.

9 Black WC, Gareen IF, Soneji SS, et al. Cost-effectiveness of CT screening in the National Lung Screening Trial. N Engl J Med 2014; 371: 1793-1802.

10 King TE, Bradford WZ, Castro-Bernardini S, et al. A Phase 3 trial of pirfenidone in patients with idiopathic pulmonary fibrosis. N Engl J Med 2014; 370: 2083-2092.

11 Richeldi L, du Bois RM, Raghu G, et al. Efficacy and safety of nintedanib in idiopathic pulmonary fibrosis. $N$ Eng J Med 2014; 370: 2071-2082.

12 Fishman A, Martinez F, Naunheim K. A randomized trial comparing lung-volume-reduction surgery with medical therapy for severe emphysema. N Engl J Med 2003; 348: 2059-2073.

13 Aberle DR, Berg CD, Black WC, et al. The National Lung Screening Trial: overview and study design. Radiology 2011; 258: 243-253.

14 Marcus PM, Gareen IF, Miller AB, et al. The national lung screening trial's endpoint verification process: Determining the cause of death. Contemp Clin Trials 2011; 32: 834-840.

15 Lynch DA, Austin JHM, Hogg JC, et al. CT-definable subtypes of chronic obstructive pulmonary disease: a statement of the Fleischner society. Radiology 2015; 277: 192-205.

16 Raghu G, Collard HR, Egan JJ, et al. An official ATS/ERS/JRS/ALAT statement: idiopathic pulmonary fibrosis: evidence-based guidelines for diagnosis and management. Am J Respir Crit Care Med 2011; 186: 788-824.

17 Pinsky PF, Kramer BS. Lung cancer risk and demographic characteristics of current 20-29 pack-year smokers: implications of screening. JNCI J Natl Cancer Inst 2015; 107: djv226.

18 Wilson DO, Weissfeld JL, Balkan A, et al. Association of radiographic emphysema and airflow obstruction with lung cancer. Am J Resp Crit Care Med 2008; 178: 738-744.

19 Le Jeune I, Gribbin J, West J, et al. The incidence of cancer in patients with idiopathic pulmonary fibrosis and sarcoidosis in the UK. Respir Med 2007; 101: 2534-2540.

20 Pritt BS, Hardin NJ, Richmond JA, et al. Death certification errors at an academic institution. Arch Pathol Lab Med 2005; 129: 1476-1479.

21 Haruna A, Muro S, Nakano Y, et al. CT scan findings of emphysema predict mortality in COPD. Chest 2010; 138: 635-640.

22 Johannessen A, Skorge TD, Bottai M, et al. Mortality by level of emphysema and airway wall thickness. Am J Respir Crit Care Med 2013; 187: 602-608.

23 Regan EA, Lynch DA, Curran-Everett D, et al. Clinical and radiologic disease in smokers with normal spirometry. JAMA 2015; 175: 1539-1549.

24 Jairam PM, van der Graaf Y, Lammers JW, et al. Incidental findings on chest CT imaging are associated with increased COPD exacerbations and mortality. Thorax 2015; 70: 725-731. 